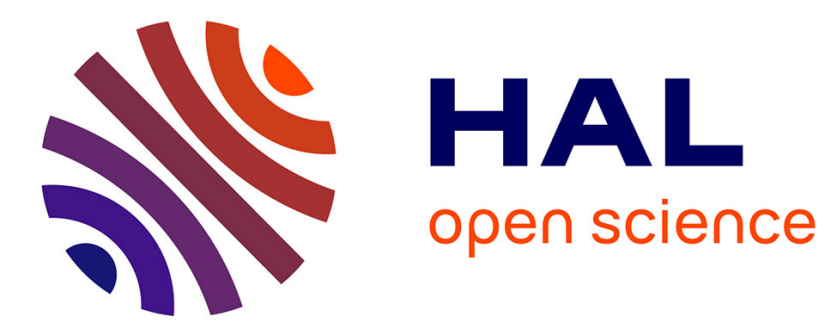

\title{
Les projets de conception comme opportunité de développements des activités
}

Flore Barcellini, Laurent van Belleghem, François Daniellou

\section{To cite this version:}

Flore Barcellini, Laurent van Belleghem, François Daniellou. Les projets de conception comme opportunité de développements des activités. Falzon Pierre (dir.). Ergonomie constructive, Presses universitaires de France, pp. 191-206, 2013, 10.3917/puf.falzo.2013.01.0191 . halshs-00860635

\section{HAL Id: halshs-00860635 \\ https://shs.hal.science/halshs-00860635}

Submitted on 10 Sep 2013

HAL is a multi-disciplinary open access archive for the deposit and dissemination of scientific research documents, whether they are published or not. The documents may come from teaching and research institutions in France or abroad, or from public or private research centers.
L'archive ouverte pluridisciplinaire HAL, est destinée au dépôt et à la diffusion de documents scientifiques de niveau recherche, publiés ou non, émanant des établissements d'enseignement et de recherche français ou étrangers, des laboratoires publics ou privés. 
Ergonomie constructive - Version finale du 22 février 2013- Ne pas diffuser

Flore Barcellini, Laurent Van Belleghem, François Daniellou

Les projets de conception comme opportunité de développement des activités

\section{Introduction}

L'ergonomie de l'activité a développé depuis une trentaine d'années une démarche d'accompagnement des projets de conception articulant analyse ergonomique du travail, démarche participative et simulation du travail. Quand certaines conditions sont réunies, cette démarche, on le constate, contribue au développement des activités et non uniquement à la conception de solutions attendues par le projet. Elle favorise ainsi l'appropriation et la mise en œuvre de ces solutions par les travailleurs, mais aussi leur maîtrise par les autres acteurs de l'entreprise. Cela contribue à renforcer à la fois le système sociotechnique et les relations sociales dans leur ensemble.

Cette dimension constructive n'est pas qu'un effet positif induit de la démarche : elle doit être considérée comme un moteur de développement de la capacité des hommes et des femmes de l'entreprise à faire face aux changements de leur situation de travail quand ils peuvent contribuer activement à sa conception. Dans ce dispositif, la simulation du travail joue un rôle central.

La dimension constructive considérée porte notamment sur :

- le développement de l'activité et des compétences des opérateurs et opératrices tout au long de la conduite de projet, permettant un début de maîtrise des futures situations avant même leur déploiement,

- le développement de l'activité des concepteurs, à travers la confrontation anticipée de leurs propositions au travail réel au cours même du processus de conception,

- le développement de la fonction décisionnelle, constituée souvent d'un ensemble d'acteurs (direction, chef de projet, ressources humaines...) devant assumer à la fois un rôle hiérarchique vis-à-vis des populations concernées par le projet et un rôle de maîtrise d'ouvrage vis-à-vis des concepteurs,

- le développement de l'activité des instances représentatives du personnel (IRP), qui peuvent trouver dans le dispositif de simulation un moyen de décaler et de restructurer les relations sociales.

Ce développement ne s'opère pas pour chacun des acteurs pris individuellement. Il se crée dans la rencontre des «mondes » (Béguin, 2007) que propose la démarche et qui nourrit les apprentissages mutuels entre ces acteurs.

Nous proposons que cette dimension constructive devienne un objectif clairement énoncé de l'intervention ergonomique en conduite de projet.

Après avoir rappelé les écueils des projets conduits avec une faible référence au travail réel, puis les principes de la démarche ergonomique de conduite de projet développée en France depuis le milieu des années 1980, ce chapitre présentera:

- les effets constatés de la simulation sur le développement de l'activité de chacun des acteurs et de leurs relations,

- l'argumentation d'un positionnement valorisant la démarche ergonomique de projet comme un processus constructif, 
- la nécessité de poursuivre l'évolution du projet initial de l'ergonomie visant l'adaptation du travail à l'être humain en l'élargissant au développement des activités.

\section{Les écueils d'une faible prise en compte du travail dans la conduite de projet}

De nombreux projets d'investissement ou de réorganisation produisent des résultats décevants, dont les symptômes habituels sont des retards au démarrage et un dépassement du budget originel dû aux mises au point nécessaires a posteriori ou encore des difficultés de maîtrise du nouveau système par les opérateurs, un taux insuffisant de fonctionnement (Wisner et Daniellou, 1984), un long délai pour atteindre le fonctionnement cible en quantité et en qualité, et parfois des accidents graves.

L'analyse de ces dysfonctionnements met souvent en évidence une double défaillance dans la conduite de projet.

- D'une part, la structure du projet elle-même est souvent en cause : faiblesse du pilotage politique du projet et de la définition des objectifs ; faiblesse de la présence de responsables d'exploitation dans le projet; portage du projet par les ingénieries mettant l'accent sur les dimensions techniques et sous-estimant celles liées aux caractéristiques de la population de travail, à l'organisation du travail, à la formation ; absence d'interactions régulières entre définition de la volonté (maîtrise d'ouvrage) et recherche des solutions (maîtrise d'œuvre) (Martin, 2000) ; caractère tardif et partiel de l'information-consultation des instances représentatives du personnel; découverte très tardive du projet par les opérateurs qui auront à agir dans le nouveau système.

- D'autre part, les ergonomes ont mis en évidence l'insuffisante prise en compte du travail humain dans les décisions de conception. Le travail qui se déroule dans les organisations antérieures au projet est souvent abordé uniquement en termes de tâches prescrites. Les régulations que les opérateurs et les opératrices mettent en œuvre pour faire face à la variabilité sont ignorées, ce qui va par exemple déboucher sur la conception d'automatismes trop simples, incapables de faire face aux situations de variabilité (Daniellou, 1987). Le travail futur, qui se déroulera dans le nouveau système, est lui aussi abordé sous l'angle des procédures prescrites, avec l'hypothèse que le travail sera une exécution de celles-ci. Les contraintes et les marges de manœuvre relatives à l'activité de travail, les conséquences sur la santé et sur la qualité de la production des biens ou des services sont peu anticipées.

Autrement dit, tout se passe comme si les prescripteurs ignoraient, dans la réflexion sur les évolutions à venir, les évolutions de l'activité. La figure 1 représente schématiquement le déroulement d'une conduite de projet sans ou avec faible prise en compte du travail réel et l'entrave que cela constitue pour le développement des activités. La situation initiale y est caractérisée par une forme d'articulation entre un système de prescription et des activités de travail qui s'influencent mutuellement (Rabardel, 1995, De Terssac, 1992). En ignorant cette articulation, les concepteurs définissent un nouveau système de prescription qui, une fois mis en œuvre, est supposé être mécaniquement "exécuté ». Les écueils énoncés ci-dessus apparaissent alors rapidement. Deux lacunes des projets de conception peuvent alors expliquer l'apparition de tensions liées à ces écueils :

- Une inadéquation du dispositif prescrit : le nouveau système de prescription (des outils, des espaces, des règles d'organisation...) tient insuffisamment compte des logiques structurantes de l'activité et de ses variabilités. Il en résulte des régulations coûteuses pour les opérateurs et opératrices, 
- Un déficit de développement: les activités utiles au fonctionnement du nouveau dispositif sont insuffisamment développées au moment de sa mise en œuvre. Il est souvent attendu que ce développement s'opère «dans l'expérience du nouveau dispositif », mais les possibilités de ce développement sont souvent entravées par l'inadéquation du nouveau système conçu aux logiques structurantes de l'activité. Les tensions perdurent.

Figure 1: Le déficit de développement des activités dans une conduite de projet sans prise en compte du travail réel

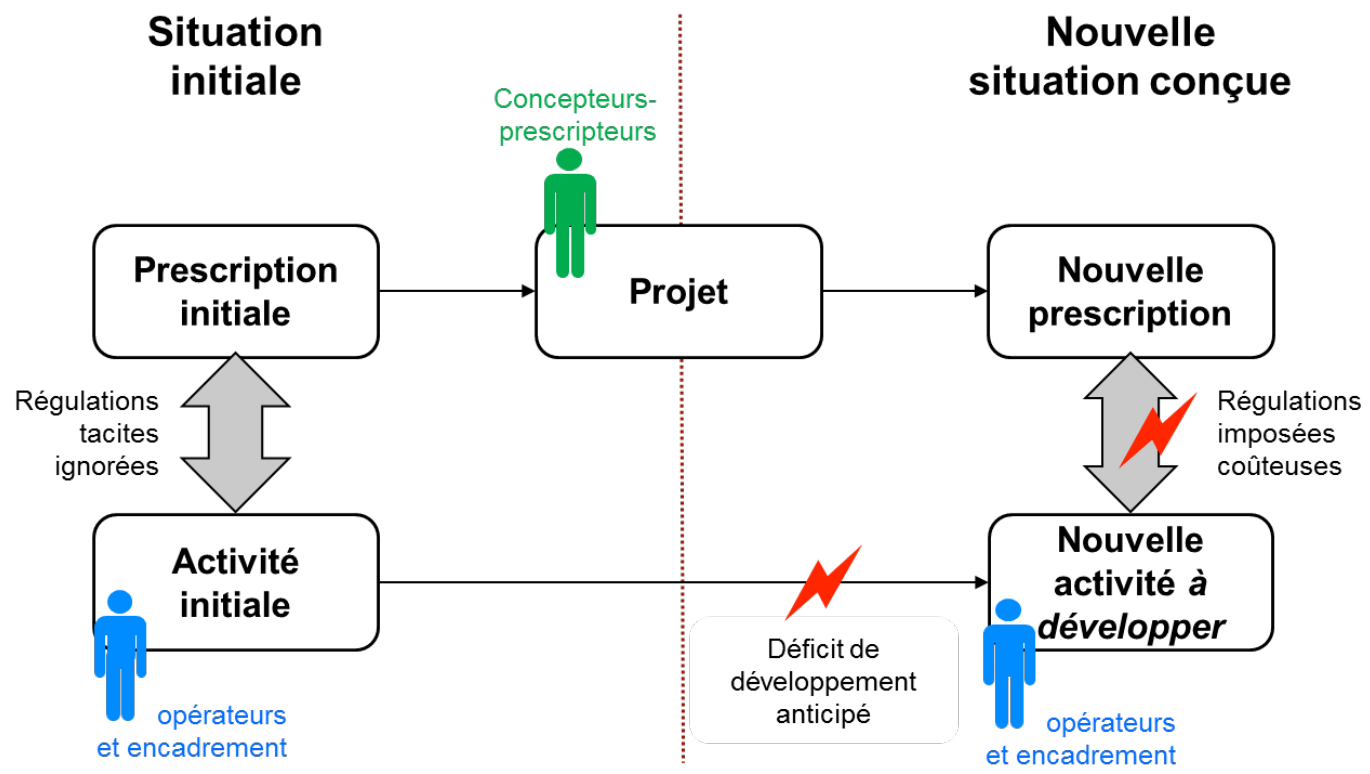

Face aux constats de l'inadéquation du dispositif prescrit, l'ergonomie de l'activité (Theureau et Pinsky, 1984 ; Daniellou, 1987 ; Maline, 1994 ; Garrigou et coll., 1995) a développé dès les années 1980 des approches de la conception, puis de la conduite de projet, destinées à favoriser une meilleure interaction entre les acteurs du projet, une meilleure prise en compte du travail antérieur et une anticipation de l'activité future. Une synthèse actualisée en est présentée ci-dessous.

\section{La conduite de projet en ergonomie : une démarche visant l'enrichissement du projet par la prise en compte du travail}

En ergonomie de l'activité, la finalité des projets de conception s'attache moins à la définition des caractéristiques des artefacts (produits, outils, espaces, postes de travail, etc.) qu'à celles des situations de travail dans lesquels ces artefacts sont présents. Dans une situation de travail, l'activité se déploie en effet dans un espace encadré par un ensemble de prescriptions : les tâches à réaliser, les espaces de travail, les équipements matériels et logiciels, la structure organisationnelle (allocation d'effectifs, formes de contrat, répartition formelle des tâches, horaires, règles...), les formations offertes... La conception de la situation de travail porte alors sur la définition de ces différentes composantes et des liens entre elles, afin de permettre le déploiement d'une activité efficace et préservant la santé des personnes. 
La conduite de projet vise à encadrer ce processus de conception au sein de l'entreprise ${ }^{1}$ et s'appuie sur une démarche :

- définie au moment du démarrage du projet,

- finalisée par la volonté de conception ou de transformation d'une ou plusieurs situation(s) de travail,

- socialement située car impliquant un collectif d'acteurs porteurs de perspectives différentes,

- structurée par une organisation et un cadre temporel et financier limité.

Cette démarche suppose d'opérer un retournement dans la relation classiquement entretenue entre ergonomes et concepteurs : l'ergonome ne doit pas se contenter d'alimenter par des recommandations des processus de conception d'artefacts maîtrisés par les seuls concepteurs ; il doit au contraire contribuer à la mise en place d'une démarche globale et structurante (Pinsky, 1992) au sein de l'entreprise, de façon à passer d'un projet conduit par la technique à une conduite de projet centrée sur le travail actuel et futur. Cette démarche permet non seulement de contribuer à la conception d'un système de travail de « qualité » mais également d'enrichir les objectifs même du projet. Les décisions de conception sont alors éclairées par des arbitrages entre les différentes dimensions de la performance (humaine, technique, économique...), leur articulation et les enjeux de santé.

Cette approche s'appuie nécessairement sur une analyse du projet et des activités de travail (portée par les ergonomes), la mise en place d'une démarche structurée, participative et concertée (favorisée par les ergonomes), la conduite de simulations du travail permettant de se projeter dans l'activité future probable (outillée et animée par les ergonomes), la formalisation des résultats des simulations en direction des acteurs du projet (concepteurs, décideurs ...) (réalisée par les ergonomes en collaboration avec ces acteurs), l'accompagnement du projet (conduit par les ergonomes) jusqu'à son démarrage.

Les acteurs devant impérativement être impliqués dans cette démarche de conception participative sont :

- Les décideurs, regroupant souvent un ensemble d'acteurs (direction, chef de projet, ressources humaines...). Porteurs des intentions du projet, ils ont le plus souvent une double fonction : ils sont à la fois engagés dans une relation fonctionnelle avec les prescripteurs (en tant que maître d'ouvrage par exemple) et dans une relation hiérarchique avec les salariés dont ils décident de transformer la situation. De ce fait, ils ont un rôle fondamental d'arbitrage entre les objectifs attendus du projet et les effets de celui-ci sur le travail réel.

- Les opérateurs et opératrices dont les activités vont être transformées dans les situations concernées par le projet (y compris l'encadrement).

- Les concepteurs, mais aussi plus globalement toute fonction interne ou externe à l'entreprise engagée dans la prescription du travail (bureau des méthodes, bureau d'études, conseil en organisation...), que nous nommerons prescripteurs.

- Les instances représentatives du personnel (IRP), qui doivent trouver toute leur place dans le dispositif mis en place.

Cette démarche est modélisée par la figure 2 (Van Belleghem \& Barcellini, 2011). Elle comporte trois étapes principales (analyser, simuler et accompagner) qui sont détaillées cidessous. La simulation de l'activité en constitue le cœur.

\footnotetext{
${ }^{1}$ Le terme « entreprise » recouvre ici toute structure employeuse (industrie, hôpital, administration...).
} 
Figure 2 : Démarche (actualisée) de conduite de projet proposée par l'ergonomie de l'activité (Van Belleghem \& Barcellini, 2011)

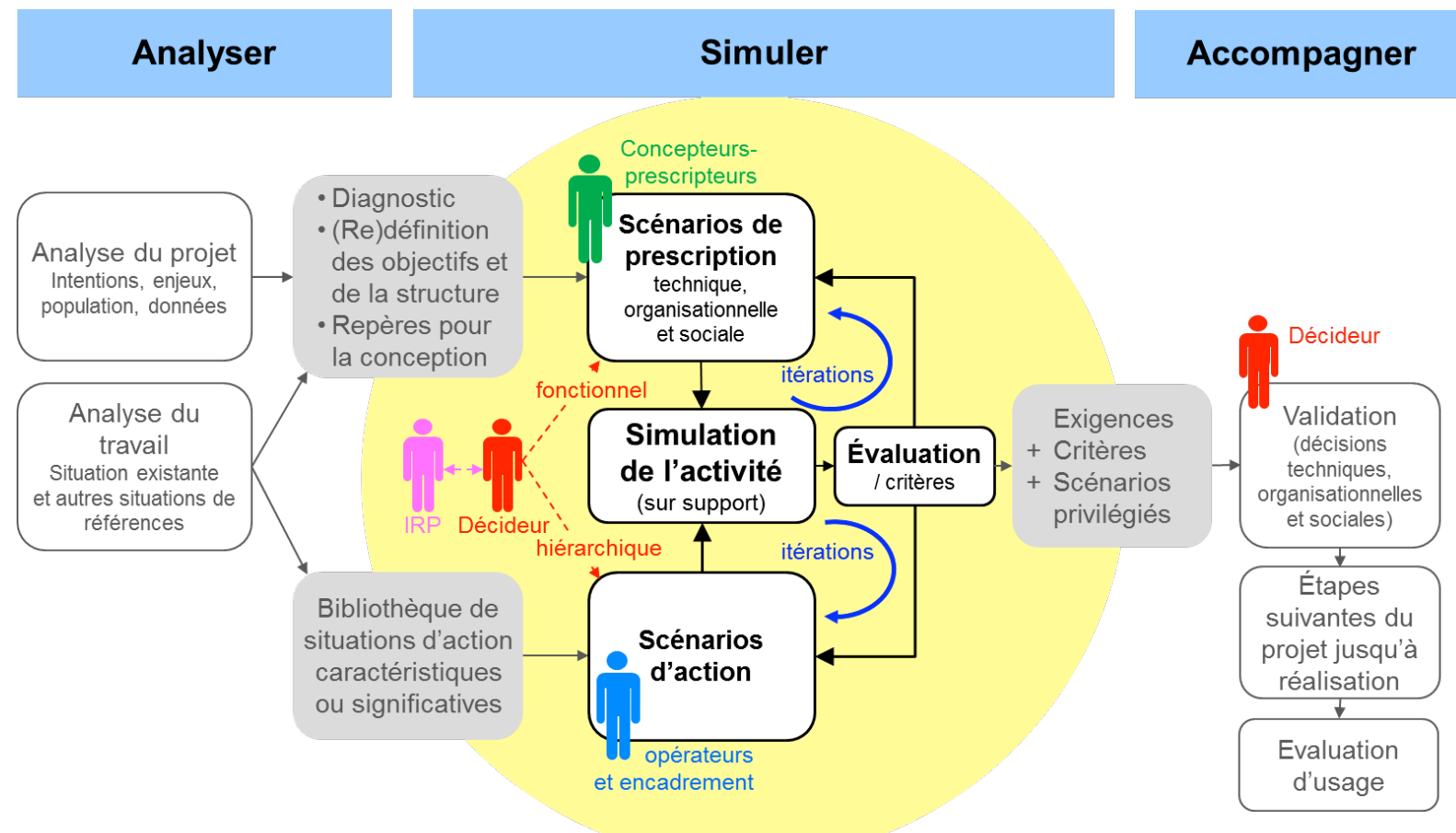

\section{Analyser : construire des connaissances sur le projet et le travail réel}

L'analyse du projet s'intéresse à ses premières intentions et à ses enjeux (économiques, de production, liés aux conditions de travail...) explicités ou non ; à la structure projet mise en place, articulant la volonté relative au future portée par la maîtrise d'ouvrage et la recherche de solutions par la maîtrise d'oeuvre ; à l'identification de la population concernée par les futures situations de travail, aux données relatives à la santé et à la performance du système. Elle permet de poser un diagnostic de projet orienté vers les décideurs et de contribuer à la structuration et à la redéfinition des objectifs du projet.

L'analyse ergonomique du travail est le premier pas de la démarche de conduite de projet. L'objectif est de produire des connaissances liées au travail qui seront utiles à l'instruction des choix du projet (aide à l'enrichissement des objectifs, de la structure et des premiers choix) et à la poursuite de la démarche (transmission de repères aux concepteurs et conduite des simulations). L'analyse $d u$ travail est conduite dans toute situation de travail, dite de référence, ayant des déterminants (techniques, organisationnels, sociaux) pertinents au regard de la situation initiale, ou de la future situation de travail. Cette analyse a plusieurs issues : elle contribue à l'enrichissement du projet, mais vise également la production de connaissances sur le travail nécessaires à la poursuite de la démarche. La formalisation de ces connaissances est dirigée :

- vers les décideurs, susceptibles de faire évoluer les objectifs du projet en fonction des constats réalisés ;

- vers les concepteurs-prescripteurs, à travers la formalisation de repères éclairant l'élaboration de premières solutions de conception (Daniellou, 2004) auxquels nous donnerons le statut de scénarios de prescription. 
- vers l'ergonome à travers la construction de bibliothèques de situations d'action caractéristiques (Jeffroy, 1987, Daniellou², 1987, Garrigou et al., 1995). Ces situations rendent compte de la variabilité des situations rencontrées par les opérateurs et opératrices et permettent d'anticiper celle à laquelle ils devront faire face dans le futur. Elles permettent d'élaborer des scénarios d'action qui seront « joués » lors des simulations.

\section{Simuler : évaluer et enrichir les propositions des concepteurs}

La simulation vise, à partir de la compréhension du travail réel actuel, à « faire jouer » par les personnes concernées le travail futur probable (sur la base de scénarios d'action) dans les conditions imposées par les nouveaux scénarios de prescriptions proposés par les prescripteurs. La simulation du travail est une méthode projective (Maline, 1994) permettant l'anticipation des conditions de réalisation de l'activité dans des conditions données. Elle permet l'évaluation des propositions des prescripteurs orientant les choix vers tel ou tel scénario de prescription, représentés par des objets intermédiaires (Jeantet et coll. 1996) et favorisant, par itération, leur amélioration progressive.

La simulation peut se dérouler suivant deux modalités principales (Daniellou, 2007) : soit grandeur nature en utilisant par exemple un prototype, les opérateurs et opératrices pouvant alors éprouver dans leur corps les améliorations apportées (ou pas) par la nouvelle solution, ce qui permet de dépasser les défenses conduisant à penser qu'aucune amélioration n'est possible (Nahon et Arnaud, 1999); soit sur un support de simulation réduit (par exemple une maquette). Dans ce second cas, l'activité peut être décrite verbalement (on parle alors de simulation langagière) mais cette description fait courir le risque d'une simulation du déroulement des tâches plutôt que de l'activité. L'usage d'un avatar de médiation de l'activité (Van Belleghem, 2012) favorise le « jeu» de la simulation, en permettant aux opérateurs et opératrices d'incarner leurs actions simulées, et contribue à la vraisemblance de la description de l'activité. La simulation nourrit le dialogue entre opérateurs et prescripteurs et autorise l'élaboration de solutions de conception négociées (e.g. Détienne, 2006, Béguin, 2007 et chapitre XXX de cet ouvrage), de compromis, dont l'expérience montre qu'ils sont parfois innovants. Ce dialogue est placé sous l'arbitrage des décideurs (en lien avec les IRP) qui doivent ouvrir ou fermer des orientations de recherche de solutions selon les objectifs visés et les moyens à engager dans le projet.

\section{Accompagner : « transformer l'essai » de la simulation}

La simulation est au cœur de la démarche ergonomique en conception. Cependant sa mise en œuvre ne suffit pas à elle seule à agir sur la situation de travail en devenir. Encore faut-il que les scénarios de prescription privilégiés soient validés par l'instance décisionnelle du projet, et qu'ils soient réellement mis en place et déployés lors du démarrage du projet. Ceci a plusieurs incidences méthodologiques. La validation par l'instance décisionnelle impose que les choix de conception, les compromis réalisés, soient justifiés au regard des enjeux du projet : les critères de choix doivent avoir été " tracés » lors des séances de simulation. La réalisation concrète des scénarios de prescription nécessite de traduire les résultats des simulations sous formes d'exigences appropriables par les concepteurs. Ces exigences formalisées sont une ressource essentielle du travail des concepteurs et permettent de progresser dans la conception $\mathrm{du}$ futur système, sa réalisation concrète, jusqu'au démarrage du projet. La démarche accompagne ce développement en mettant en place de manière itérative des simulations de

\footnotetext{
${ }^{2}$ Dans cet article, les situations d'action caractéristiques étaient appelées «actions-types », terme qui a été corrigé par la suite sur la base des travaux de Jeffroy (1987).
} 
plus en plus détaillées qui permettent de raffiner le système à concevoir, jusqu'à son démarrage.

La démarche présentée a été mise en œuvre un grand nombre de fois depuis sa formalisation initiale et porte aujourd'hui non seulement sur la conception de systèmes techniques, mais aussi sur la conception d'organisations du travail (Carballeda \& Daniellou, 1997; Petit et coll., 2011; Van Belleghem, 2012). A ces occasions, on a pu constater des formes de développement de l'activité, notamment dans les phases de simulation, qui, selon nous, compensent la lacune liée au déficit de développement (identifiée en section 1). Considéré jusqu'alors comme un effet induit de la démarche, ce développement peut être envisagé comme une finalité de la démarche à part entière.

\section{Le projet de conception comme opportunité de développement des activités}

La mise en œuvre de la démarche décrite ci-dessus produit des effets dépassant fréquemment les objectifs initiaux portés par le projet. On observe notamment que la participation outillée des acteurs à cette démarche contribue autant à la conception de la situation future qu'au développement de leur activité, au cours même de la démarche. Elle contribue aussi au développement des activités des autres acteurs engagés dans la démarche: décideurs, concepteurs, représentants du personnel. De ce fait, quand les conditions sont réunies, elle renforce ces fonctions et les relations professionnelles entre elles tant au cours du projet (coopération dans le projet) qu'après sa mise en œuvre opérationnelle (coopération dans le travail). On constate alors que la réussite du projet tient autant à la pertinence des choix réalisés dans le projet qu'à la qualité du processus de développement qu'il a permis.

Les mécanismes de développement observés sont détaillés ci-dessous par type d'acteurs.

\section{Le développement de l'activité des opérateurs et opératrices}

La démarche proposée inscrit une situation d'activité particulière, la situation de simulation (Béguin et Weill-Fassina, 1997), entre la situation d'activité antérieure et la nouvelle situation conçue. Cette situation de transition offre l'opportunité d'un développement des activités des futurs «utilisateurs» du système (différents opérateurs et opératrices et encadrement de proximité principalement) en amont de la mise en œuvre du projet, tout en contribuant à sa conception. La figure 3 en schématise la progression.

Figure 3 : la situation de simulation, creuset du développement des activités 


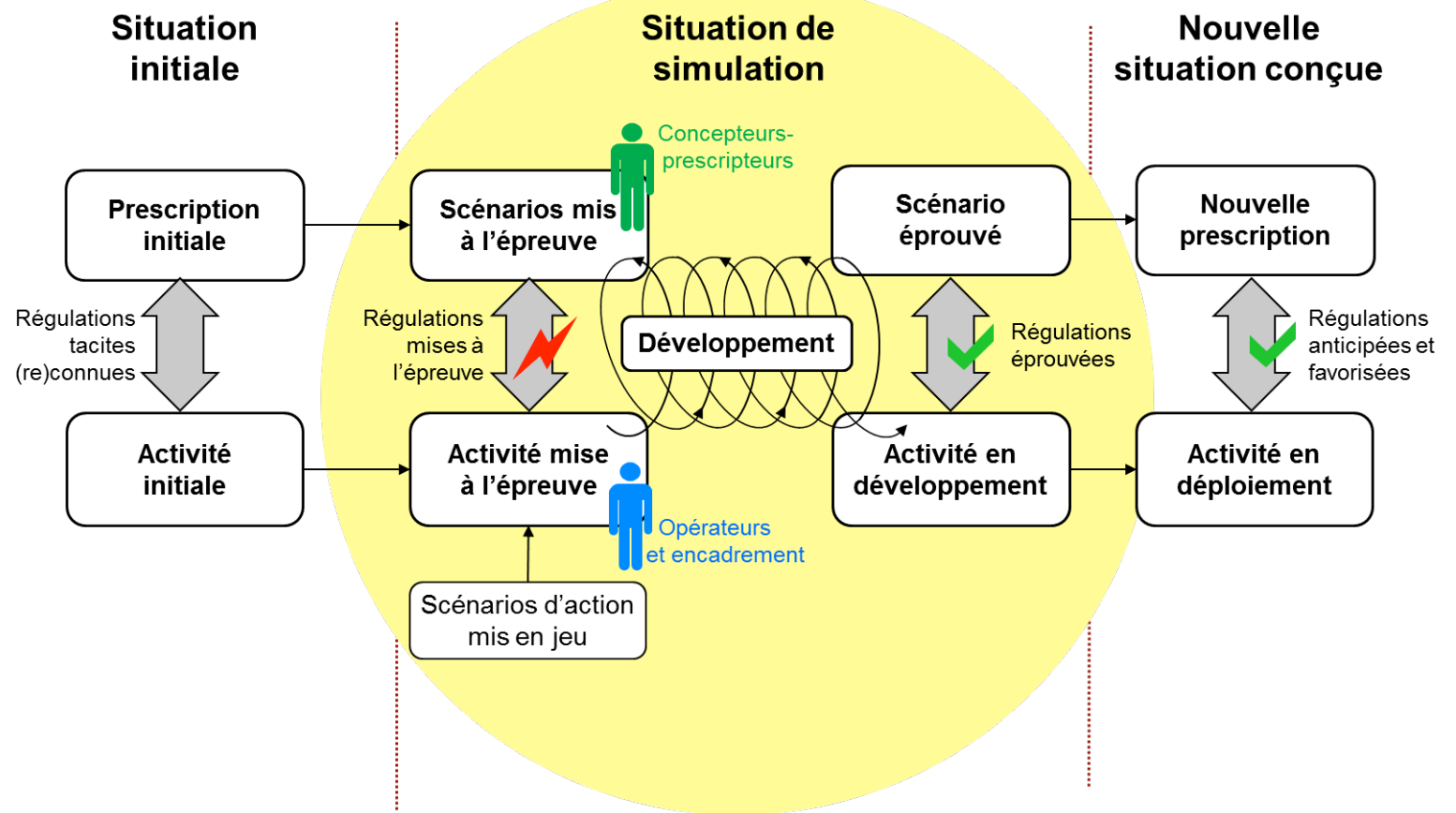

Nous l'avons dit, la simulation vise à faire jouer leur travail futur probable à des opérateurs et opératrices sur la base des scénarios de prescription proposés par les concepteurs et de scénarios d'action issus de l'analyse de situations existantes. C'est une mise à l'épreuve du scénario de prescription autant que de l'activité. Dans un premier temps, cette mise à l'épreuve fait toujours apparaître des difficultés, voire des impasses ou des désaccords (Béguin, 2013), dans la réalisation de l'activité simulée. Ces difficultés n'ont pas de conséquences graves, car elles émergent avant réalisation des solutions (justifiant l'usage du terme "scénario", qui, lui, autorise des évolutions). Au contraire leur mise en évidence précoce (à l'inverse de ce qui se passe, trop souvent, dans les processus de conception " classiques ») révèle des lacunes qui conduiraient à des difficultés de fonctionnement ultérieures, évitables par des modifications des scénarios de prescription. Mais ces difficultés peuvent aussi révéler des nécessités de développement de l'activité pour que les opérateurs et opératrices puissent mieux s'approprier les principes de prescription s'avérant pertinents. La simulation doit permettre, là aussi, de mettre à l'épreuve les modalités de cette appropriation. Le scénario peut être éventuellement adapté pour favoriser ce processus de développement. Ainsi menée, la démarche conduit à concevoir un système de prescription en fonction du développement de l'activité qu'il permet.

Ce double mouvement de développement de la prescription et de l'activité s'apparente au processus d'instrumentalisation/instrumentation proposé par Rabardel (1995), s'inspirant luimême du processus d'accommodation/assimilation de Piaget.

Les itérations de ce double mouvement, s'appuyant sur une large gamme de situations d'action caractéristiques, mènent à l'élaboration d'un scénario de prescription suffisamment stabilisé après des mises à l'épreuve successives. Mais elles mènent aussi à l'élaboration de nouvelles formes d'activité, éprouvées par les opérateurs et opératrices participant à la démarche, avant même la mise en application concrète du projet. Au moment de celle-ci, l'activité qui a commencé à se développer pendant les simulations pourra alors se déployer dans le nouveau système en poursuivant son développement. A la veille de la mise en œuvre d'une nouvelle installation, d'un nouveau logiciel ou d'une nouvelle organisation, il n'est pas rare d'entendre les opérateurs et opératrices ayant participé à la démarche manifester leur 
impatience à « y être déjà » et évoquer leur confiance dans la réussite du projet - ce constat tranche avec de nombreuses conduites de projet, où des formes de résistance et de méfiance sont régulièrement observées. Mais la simulation portant sur plusieurs scénarios de prescription (pas simplement celui qui sera retenu au final), elle s'étend sur un espace d'exploration et d'expérimentation large de manières de faire et favorise leur mise en débat, voire en controverse, non seulement entre opérateurs et prescripteurs, mais aussi entre les opérateurs et opératrices eux-mêmes. Or, c'est bien ce type de mise en débat, portant notamment sur les critères de la qualité du travail, que Clot (2011) appelle de ses vœux pour poser les bases du « bien-faire », sans lequel aucun « bien-être » n’est possible.

La simulation contribue ainsi à la construction d'une expérience riche des différents scenarios explorés, élargissant progressivement la " gamme des gestes » professionnels possibles (Clot, ibid. p.102). Même si l'activité développée en simulation continuera son développement en situation réelle, elle couvre parfois un champ des possibles plus large que ce que demandera la nouvelle situation. La situation de simulation comporte en effet de multiples « essaiserreurs » arbitrés collectivement qui contribuent à nourrir l'activité en développement.

Pour éviter un décalage entre les opérateurs ayant participé aux simulations et les autres, il est nécessaire de concevoir un dispositif complémentaire de formation s'appuyant là encore sur la mise en œuvre de simulations du travail, mais autour du seul scénario éprouvé et validé. On attendra de cette formation un développement des activités « d'usage » du futur système avant même sa mise en place opérationnelle.

\section{Le développement de l'activité des concepteurs}

Les concepteurs sont invités à participer activement à la simulation. Il est attendu d'eux qu'ils proposent des scénarios de prescription initiaux (et pas seulement une solution unique) s'appuyant sur les repères issus du diagnostic, qu'ils assistent aux simulations réalisées par les opérateurs, qu'ils discutent et argumentent leurs critères d'évaluation et qu'ils fassent évoluer les scénarios vers une meilleure intégration de l'activité en développement. A cette occasion, on constate un développement de l'activité des concepteurs.

Généralement impliqués dans un processus à dominante technique, centré sur la seule composante matérielle ou procédurale du système à concevoir, les concepteurs sont ici confrontés à l'activité des opérateurs et à ses possibilités de développement. Rendue visible par la simulation, cette activité peut leur apparaître au départ comme une contrainte supplémentaire, encombrante, qu'il leur serait plus facile d'ignorer. Mais elle devient rapidement l'enjeu de la conception dès lors qu'elle se révèle être la condition même du fonctionnement du système.

Un retournement s'opère alors : de contrainte qu'elle était, l'augmentation des exigences de conception liée à la reconnaissance de l'activité réelle de travail devient ressource pour les concepteurs. En les outillant pour imaginer de nouvelles solutions pour mieux répondre au débat de critères qui s'engage entre les participants (par exemple entre l'exigence de productivité et l'exigence de qualité), elle constitue pour eux une source d'innovation (au sens où l'innovation est appropriation) en élargissant le champ des possibles. Si la tâche, comme le souligne Clot (1999), est le produit refroidi de l'activité des concepteurs, il y a un enjeu à mettre cette activité " tant qu'elle est encore chaude» à l'épreuve de l'activité en développement des opérateurs. C'est dans ce creuset que constitue la démarche participative, chauffé à blanc par la simulation du travail, que les apprentissages mutuels (Béguin, 2007 et 
chapitre XXX de cet ouvrage) entre concepteurs et opérateurs prennent toute leur force... et contribuent au développement de l'activité des concepteurs.

L'engagement dans cette démarche implique que l'activité de conception soit distribuée et que les concepteurs acceptent de partager une partie de la conception, notamment en ce qui concerne :

- l'élaboration des scénarios de prescription, pour lesquels chaque participant (opérateurs, encadrement, décideur, IRP) peut être force de proposition,

- l'élaboration des critères d'évaluation du système conçu, qui ne renvoient plus seulement à la cohérence technique du système, mais sont complétés par des critères liés au développement d'une activité de travail efficace, de qualité, et produisant $\mathrm{du}$ sens.

Il est paradoxal, de ce point de vue, de constater que le développement de l'activité des concepteurs est conditionné à une "dépossession» d'une partie de celle-ci. La situation de simulation semble offrir ici l'occasion d'une réflexivité du concepteur sur sa propre pratique (Schön, 1983), qui l'aide à orienter son activité vers une plus grande pertinence du système conçu, quitte à l'éloigner de ses modèles initiaux. Cela peut contribuer à renouveler le sens du métier des ingénieurs, mis à mal par les lacunes des conduites de projet (Charue-Duboc et Midler, 2002).

\section{Le développement de l'activité des décideurs}

La démarche contribue à un développement de la fonction décisionnelle. Elle permet aux décideurs de réaliser la primauté - et donc la responsabilité - de la fonction de maîtrise d'ouvrage qui fixe une volonté pour le futur, par rapport à celle de la maîtrise d'œuvre, dont la fonction est de trouver des solutions pour mettre en œuvre cette volonté. Les écarts entre « ce qui est souhaité » et « ce qui est faisable » nécessitent des arbitrages, par ajustement des objectifs ou des ressources. Cette prééminence doit s'exercer sur toutes les dimensions (technique, organisation, formation) pendant toute la durée du projet, ce qui suppose une organisation de l'activité de décision :

- d'une part, un collectif de responsables, représentant les différentes logiques vitales pour le développement de l'entreprise (finances, marketing, ressources humaines, qualité, sécurité, environnement...),

- d'autre part une représentation permanente de ce collectif par un chef de projet qui assure l'interface quotidienne avec la maîtrise d'œuvre.

Le développement de l'activité de décision passe notamment par un renforcement de la conscience de la diversité des logiques à prendre en compte, des contradictions entre elles, et de l'intérêt d'une construction collective de compromis au sein de l'équipe de direction. En mettant les prescriptions techniques et organisationnelles à l'épreuve de la simulation du travail, la démarche ergonomique contribue à instaurer une force de rappel par la réalité et à éviter la construction de «défenses gestionnaires » du type « de toute façon ça marchera!» Elle fait émerger de possibles coûts cachés et des conflits de critères. Mais elle contribue aussi, parfois, à permettre l'ouverture de nouvelles perspectives d'organisation et de gestion, qui seraient apparues incongrues ou inaccessibles tant que leur viabilité n'a pas été mise en lumière par une simulation.

Ce constat peut conduire les décideurs à modifier leur stratégie d'annonce pour les projets ultérieurs : plutôt que d'attendre, pour l'annoncer, que le projet soit stabilisé, ils considèrent 
l'annonce précoce de projets encore incertains comme une occasion positive d'enrichir ceuxci par la mise en débat à la lumière des possibilités de l'activité.

La démarche ergonomique en conduite de projet contribue aussi à renforcer le « droit au jeu » des opérateurs et opératrices et de l'encadrement de proximité comme interlocuteurs de la conception. La découverte, par les décideurs, que cette contribution a permis d'éviter des erreurs majeures peut favoriser l'instauration de nouvelles pratiques gestionnaires. Il en va de même pour les relations avec les instances représentatives du personnel.

\section{Le développement de l'activité des IRP}

La démarche ergonomique décrite ci-dessus constitue également une épreuve et une opportunité pour les instances représentatives du personnel (IRP). Epreuve, car elle peut venir interpeller une culture de représentation par délégation, où les représentants du personnel se considèrent comme les uniques porteurs légitimes du point de vue des salariés sur leurs conditions de travail. Opportunité aussi, car les résultats de l'analyse de l'activité dressent une image de salariés non seulement contraints par leur environnement de travail, mais pleinement engagés dans la poursuite de ce qu'ils considèrent comme du travail bien fait (Chassaing et coll., 2011), et porteurs de savoirs insoupçonnés. Dans certains cas, ce renversement a conduit des organisations syndicales à modifier explicitement leurs pratiques de relations aux salariés, en cherchant à les baser sur la compréhension du travail.

La démarche peut contribuer au développement de l'activité des IRP quand des enjeux organisationnels importants sont présents, en lui offrant deux outils majeurs: la compréhension détaillée de l'activité existante, et la réflexion sur le futur en termes de conséquences sur le travail. Un processus structuré d'instruction des choix peut ainsi se développer, en lieu et place des confrontations rituelles antérieures (Dugué, 2008). Le « traçage » des décisions de conception au fur et à mesure de leur formalisation permet aussi aux IRP, le cas échéant, de rappeler leur importance si elles étaient oubliées à des phases de réalisation ultérieures.

Ce développement de l'activité des IRP autour de la question du «travail » n'empêche pas qu'elles ont à prendre en compte beaucoup d'autres dimensions que celles qui font l'objet des simulations ergonomiques: l'emploi, les salaires, les statuts, etc. Dans certains cas, on constate l'irrigation de ces dimensions « macro» par la réflexion sur le travail développée dans le projet : c'est le cas par exemple quand les négociations sur les effectifs utilisent les scénarios d'action proposés pour les simulations afin que le personnel soit suffisant pour faire face non seulement aux situations normales, mais aussi aux situations incidentelles.

\section{Conclusion : De l'adaptation du travail au développement de l'activité}

La reconnaissance d'un développement des activités au sein de la démarche ergonomique de conduite de projet (et plus largement dans toute situation de travail) participe d'une nouvelle évolution du projet fondateur de l'ergonomie visant l'«adaptation du travail à l'être humain ». Cette visée s'est traduite initialement dans les orientations originelles de l'Ergonomics Research Society en 1949, sous l'expression "fitting the job to the worker », par la recherche d'une adéquation entre le travail et les caractéristiques psychologiques et physiologiques de l'être humain. Cette orientation s'est notamment traduite par ce que Hubault \& Bourgeois (2004) appellent « l'ergonomie de la tâche », avec le développement de 
prescriptions ergonomiques directement applicables dans la conception des moyens de travail $^{3}$.

En parallèle s'est développée une "ergonomie de l'activité » (notamment dans les pays francophones, mais aussi scandinaves ou sud-américains) mettant l'accent sur la contribution active de l'opérateur à l'accomplissement des tâches compte tenu des inéluctables variabilités des situations réelles. Cette ergonomie de l'activité, initialement centrée sur la compréhension du travail, s'est orientée dans les années 1980 vers la prise en compte du travail réel dans la conduite de projet, comme cela a été décrit plus haut. L'enjeu est alors de concevoir des espaces pour l'activité future (Daniellou, 2004) laissant des possibilités de marges de manœuvre, de gestion de leur activité aux opérateurs et aux opératrices, voire des possibilités de conception continuée dans l'usage (Rabardel, 1995).

La perspective de l' « ergonomie constructive » est alors d'élargir la notion d' « adaptation » à celle de développement de l'activité : l'objectif de l'ergonome est de contribuer à la conception de situations de travail servant de point d'appui au développement de l'activité des hommes et des femmes partie prenantes du projet. Faire débuter ce développement dès la conception des situations de travail, faisant du processus de construction de l'expérience (par la simulation du travail) le critère d'évaluation des solutions produites par les concepteursprescripteurs, peut être un choix stratégique pour l'ergonome.

Cette perspective appelle sans doute le renforcement de programmes de recherche, à la fois sur les apprentissages à l'œuvre chez les différents acteurs impliqués dans la conduite de projet (dont l'ergonome), et sur les méthodes et pratiques d'intervention permettant de soutenir ce développement de leurs activités.

\section{Bibliographie}

BEGUIN, P. (2007) Innovation et cadre sociocognitif des interactions concepteursopérateurs : une approche développementale. Le Travail Humain, 70(4), 369-390.

BEGUIN, P. et WEILL-FASSINA, A. (1997) De la simulation des situations de travail à la situation de simulation. La simulation en ergonomie: connaître, agir et interagir. Toulouse : Octarès.

CARBALLEDA, G. \& DANIELLOU, F. (1997) Ancrer le changement de l'organisation dans une compréhension du travail actuel. Educations n ${ }^{\circ} 13$, p.50-55.

CHARUE-DUBOC, F. et MIDLER, C. (2002). L'activité d'ingénierie et le modèle de projet concourant. Sociologie du Travail, 44(3), 401-417.

CHASSAING, K., DANIELlOU, F., DAVEZIES, P. et DURAFFOURG, J. (2011). Recherche action «Prévenir les risques psychosociaux dans l'industrie automobile: élaboration d'une méthode d'action syndicale». Emergences-CGT-Ires, 2011

CLOT, Y. (1999). La fonction psychologique du travail. Paris : PUF.

CLOT, Y. (2011). Le travail à cour : pour en finir avec les risques psycho-sociaux. Paris : La découverte.

DANIELLOU, F. (1987) Automatize What : Facts or Fiction ?, in : WISNER, A., (Ed.) New Techniques and Ergonomics, 326 p., Paris : Hermès Pub.

\footnotetext{
${ }^{3}$ Cette dynamique s'est développée aux Etats-Unis sous le nom de Human Factors et au Royaume-Uni sous celui d'Ergonomics. La convergence entre les deux termes s'est traduite par le changement de nom de la société américaine de Human Factors Society en Human Factors and Ergonomics Society en 1992, et par la transformation en 2009 de l'Ergonomics Society britannique en Institute of Ergonomics and Human Factors.
} 
DANIELLOU, F., (1987) Les modalités d'une ergonomie de conception, son introduction dans la conduite des projets industriels, Note documentaire ND 1647-129-87, Paris : INRS.

DANIELLOU, F., (2004), L'ergonomie dans la conduite de projets de conception de systèmes de travail, pp. 359-373 in P. Falzon, Ergonomie, Paris : PUF

DANIELLOU, F., (2006), Entre expérimentation réglée et expérience vécue, les dimensions subjectives de l'activité de l'ergonome en intervention, Activités, 3, 1, 5-18

DANIELLOU, F., EKLUND, J. (1991) Ergonomics and project management 2 : different approaches for different phases of the project, pp 1332 -1334, in Quéinnec, Y., Daniellou, F., (Eds), Designing for Everyone, Actes du XI ${ }^{\circ}$ Congrès de l'IEA, Taylor et Francis, Londres.

DANIELlOU, F., GARRIGOU, A. (1992) Human Factors in Design : Sociotechnics or Ergonomics ? pp 55-63, in Helander, M., Nagamachi, M. (Eds), Design for Manufacturability. A systems approach to concurrent engineering and ergonomics, Taylor and Francis, Londres.

DETIENNE, F. (2006). Collaborative design: Managing task interdependencies and multiple perspectives. Interacting with Computers, 18(1), 1-20.

DUGUE, B., (2008), Les paradoxes de la participation du CHSCT dans la conduite des projets de conception, pp. 49 à 53, Actes du 43e congrès de la Société d'Ergonomie de Langue Française : Ergonomie et conception. Toulouse : Octarès Editions.

EKLUND, J., DANIELLOU, F., 1991, Ergonomics and project management 1 : important aspects in the planning of the project, pp 1329 -1331, in Quéinnec, Y., Daniellou, F., (Eds), Designing for Everyone, Actes du $\mathrm{XI}^{\circ}$ Congrès de l'IEA. Londres : Taylor et Francis.

GARRIGOU, A, DANIELlOU, F., CARBALlEDA, G. et RUAUD, S. (1995). Activity analysis in participatory design and analysis of participatory design activity. International Journal of Industrial Ergonomics, 15, 311-327.

HUBAULT, F., BOURGEOIS, F. (2004), Disputes sur l'ergonomie de la tâche et de l'activité, ou la finalité de l'ergonomie en question, Activités, 1, 1, 34-53

JEANTET, A., TIGER, H., VINCK, D., TICHKIEWITCH, S.(1996) La coordination par les objets dans les équipes intégrées de conception, pp 87-100, in G. de Terssac et E. Friedberg (dir.), Coopération et conception, Toulouse : Octarès Éditions.

JEFFROY, F. (1987) Maîtrise de l'utilisation d'un système micro-informatique par des utilisateurs non informaticiens. Thèse de doctorat en ergonomie, Paris : Cnam.

MALINE, J. (1994). Simuler le travail. Lyon : Editions de l'ANACT.

MARTIN, C. (2000) Maîtrise d'ouvrage, maîtrise d'œuvre, construire un vrai dialogue. La contribution de l'ergonome à la conduite de projet architectural. Toulouse : Octarès Éditions. NAHON, P., ARNAUD, S. (1999). Sortir de la boucle infernale : essai de maitrise dans trois abattoirs de porcs. Actes du $34^{e}$ congrès de la SELF, Caen, 15-17 Septembre 1999, pp. 147153.

PETIT, J., Dugué, B. et Daniellou, F. (2011). L'intervention ergonomique sur les risques psychosociaux dans les organisations : enjeux théoriques et méthodologiques. Le Travail Humain, 74(4), 391-409.

PINSKY, L. (1989). De l'interface à la situation de travail, in V. De Keyser \& A. Van Daele, L'ergonomie de conception, Liège : De Boeck Université.

PINSKY, L. (1992). Concevoir pour l'action et la communication, Essais d'ergonomie cognitive. Présentation et postface de J. Theureau. Berne : Peter Lang

RABARDEL, P. (1995) Les hommes et les technologies, approche cognitive des instruments contemporains, Paris, Armand Colin.

SCHON, D.A. (1983). The reflective practitioner: How practitioners think in action. London, UK : Temple Smith.

TERSSAC (de), G. (1992) Autonomie dans le travail. Paris : Presses Universitaires de France. 
THEUREAU, J., PINSKY, P. (1984). Paradoxe de l'ergonomie de conception et logiciel informatique. Revue des Conditions de Travail, 9, 25-31.

VAN BELLEGHEM, L. \& BARCELLINI, F. (2011). Cours ergonomie et conception : modèles et outils pour l'action. Cnam, Paris.

VAN BELLEGHEM, L. (2012). Simulation organisationnelle : innovation ergonomique pour innovation sociale. In Actes du 42 $2^{\text {ème }}$ congrès de la SELF, 05-07 Septembre, Lyon, France.

WISNER A. et DANIELLOU, F. (1984). Operation rate of robotized systems : the contribution of ergonomic work analysis, in HENDRICK, H.W., \& BROWN., O. Jr, Human Factors in Organizational Design and Management, pp 461-465, Elsevier Science Publishers B.V., Amsterdam 